\title{
A Bragg-like chirped clad all-solid microstructured optical fiber with ultra-wide bandwidth for short pulse delivery and pulse reshaping
}

\author{
Somnath Ghosh ${ }^{(1)}$, R. K. Varshney ${ }^{(1)}$, Bishnu P. Pal ${ }^{*(1)(2)}$, and Gérard Monnom ${ }^{(2)}$ \\ ${ }^{(1)}$ Department of Physics, Indian Institute of Technology Delhi, New Delhi 110016, INDIA \\ (2) Laboratoire de Physique de la Matière Condensée, Université de Nice-Sophia Antipolis, \\ CNRS UMR 6622, 06108 Nice Cedex 2, FRANCE \\ *Tel: +91-11-26591331, Fax: +91-11-26581114, e-mail: bishnupal@gmail.com
}

\begin{abstract}
Chirped cladding is proposed as a novel tailoring tool to simultaneously attain wider transmission window and reduced temporal dispersion in an all-solid Bragg-like microstructured optical fiber as compared to its perfectly periodic cladding counterpart. This design route for photonic bandgap microstructured fibers could be exploited as an additional degree of freedom for bandgap engineering. A suitably chirped clad fiber could be gainfully exploited to deliver femto-second pulse with ultra wide bandwidth. Further, generation of self-similar parabolic profile pulse is demonstrated by propagating an input Gaussian pulse through such a 2 meter long linearly tapered Bragg like fiber sample.
\end{abstract}

Keywords Microstructured fiber; Bandgap engineering; Short pulse delivery; Parabolic pulse generation

\section{Introduction}

Light guidance through photonic bandgap (PBG) microstructured optical fibers (MOF) has emerged as a research field of intense contemporary interest (Knight et al. 1998; Russell 2003; Temelkuran et al. 2002). In addition to the freedom offered by photonic bandgap structures through a multitude of structural parameters to tailor their loss and dispersion characteristics, light could be also guided through even sharp bends in such structures. These attractive features of photonic bandgap structures have indeed led to a large number of proposals for their potential applications in areas like integrated optical devices, biomedical optics, and mid-IR photonics (Liu et al. 2008; Nielsen et al. 2006; Millo et al. 2008). Due to the ease of tailoring their nonlinearity and dispersion, these fibers are also attractive for pulse reshaping, supercontinuum generation, pulse compression, parabolic pulse generation and several other nonlinear applications (Hirooka and Nakazawa 2004; Ranka et al. 2000; Pal et al. 2006; Nagaraju et al. 2010). Distortion less propagation of short pulses of tens-of-femto second duration through the MOFs are fundamental requirement for these applications. Also in medical applications, which involve delivery of large intensity optical pulses, one requires undistorted propagation (i.e. propagation with minimal dispersion as well as loss) of very short pulses through the designed fiber length. To fulfill this goal, it is important to operate the geometry at the center of its bandgap, (because the device otherwise could suffer from excessive loss) while maintaining low dispersion. Thus any bandgap engineering tool, which enables wavelength tunablity of the center of the bandgap in photonic bandgap guided devices, should be attractive for certain applications. Recently, it is reported that introduction of a certain amount of aperiodicity across the cladding layers in their spatial and/ or refractive index periodicity in the transverse plane could significantly modify the propagation characteristics of microstructured fibers and waveguides (Pal et al. 2007; Skibina et al. 2008; Pal et al. 2009). This effect could thus be exploited as a design tool to tailor their propagation characteristics (Mori and Baba 2004; Saitoh et al. 2003; Li et al. 2007).

In the present paper, we report for a new photonic bandgap guided all-solid Bragg-like fiber structure, which could be fabricated by the well known extrusion technique. In our sample design, we assumed the Bragg-like fiber to be based on soft glasses as the host, and its central core region is surrounded by a cladding that is linearly chirped in the spatial (radial) direction 
and/or in the refractive index values of the cladding layers. Our choice of the glass system is driven by the fact that it is all-solid and also such a glass system could supposedly yield reproducible MOFs with parameters very close to the designed values (Feng et al. 2005; Price et al. 2007). We demonstrate that by introducing a suitably chosen aperiodicity in the claddings one could attain a much wider photonic bandgap with a significantly reduced dispersion as the most important benefit. Through tapering the proposed chirped microstructured fiber along its length, we have introduced an additional degree of freedom to tailor their nonlinearity and dispersion characteristics. These features yield a new platform for pulse reshaping. As an example, we demonstrate generation of parabolic pulse from a tapered all-solid Bragg-like chirped microstructured optical fiber by propagating a Gaussian pulse. It is also shown that this new passive route of parabolic pulse generation minimizes potential instabilities due to third order dispersion (TOD) and finite loss of the device.

\section{Theory and modeling}

PBG guided flow of light through a microstructured geometry is controlled by the spatial and refractive index periodicity of the surrounding cladding layers. The characteristic bandwidths of such structures are dictated by the unit cell specifications of their multilayer claddings. Whereas in the case of our proposed chirped Bragg-like fiber geometry (CBLF) (schematically shown in Fig. 1(a) along with its refractive index profile in Fig. 1(b)), it is possible to suitably engineer the bandgap for purposeful designs through appropriate choice of cladding periods. For example, the very first bi-layer adjacent to the central core inherits a characteristic bandwidth (for light guidance through the core), which gets modified by the subsequent deliberately chosen aperiodic bilayers. As a result, the overall structure exhibits Bragg resonances in a distributed manner across a wider frequency band and hence yields less selectivity to the operating wavelength. In Fig. 1(a) we have designated the wavelength range guided by the bandgap of first bi-layer of the chirped cladding as $\Delta \lambda_{1}$. Here it is assumed that we have infinite number of only such periodic bi-layers surrounding the core. Similarly the successive bilayers of different periodicities guide wavelength range $\Delta \lambda_{2}$ and $\Delta \lambda_{3}$ respectively. Thus the wavelength range effectively guided by the core is an overlap of all these windows. We designate the thicknesses of the first cladding bi-layer adjacent to the core as $\left(d_{1}\right)_{\text {initial }}$ and $\left(d_{2}\right)_{\text {initial }}$, respectively, and those corresponding to the final cladding bi-layer as $\left(d_{1}\right)_{\text {final }}$ and $\left(d_{2}\right)_{\text {final }}$. In between these two terminal bi-layers, the cladding thickness of the intermediate bi-layers is assumed to be linearly up-/down-chirped till the targeted value of the final bi-layer is achieved. One can define a chirp factor as

$$
f_{\mathrm{j}}=\frac{\left(d_{\mathrm{j}}\right)_{\text {final }}}{\left(d_{\mathrm{j}}\right)_{\text {initial }}} ; \quad(\text { for } \mathrm{j}=1,2)
$$

So, in a chirped photonic cladding geometry, we have coaxial bilayers with slightly varying (a perturbation from the underlying periodic structure) refractive index/ spatial co-ordinate specifications surrounding the core. The characteristics band diagrams for the first bilayer, assuming that its own periodicity remains constant over the finite cladding and that for the periodicity of the next bilayer is obtained. It is evident that (due to slight variation in periodicity) there is a common stop band for these two band plots of successive bilayers. But the upper and lower cut-off wavelengths are different. Certainly, these two bilayers will help to confine light of wider bandwidth than the bandwidth of individual periods. This argument valid for all other bilayers of bandgap transition in a constantly varying periodicity makes the chirped photonic cladding light guiding geometries very interesting. Figure 2(a) shows band diagrams for the innermost and outermost bilayers for a particular chirp factor $\left(f_{1}=1.5\right.$ and $f_{2}$ $=1$ ) of the entire cladding. Whereas, Fig. 2(b) is an enlarge view over a narrower frequency window of the fundamental bandgap marked in Fig. 2(a). This figure along with Fig. 2a, illustrate a continuous variation of the stop band frequency range; wherefrom it is evident that one could exploit this route to enlarge the width of the photonic bandgap. The enhancement of the stop band frequency range is controlled by the chirp parameter as defined by Eq. (1). We have observed that if the spatial dimensions of the high and low index layers are varied in 
two different proportions (i.e., values of $f_{1}$ and $f_{2}$ are different), this chirping tool becomes more effective to achieve substantial widening of the transmission window and elimination of the highly dispersive strong resonances in an otherwise PBG guided structure. In order to get a quantitative appreciation of this feature, we have assumed no variation in the optogeometric parameters of the fiber along its longitudinal direction. For the material constituents, we chose soft glasses SF6 (refractive index $\left(n_{1}\right)=1.7641$ at wavelength $\lambda=1550 \mathrm{~nm}$ ) and LLF1 (refractive index $\left(n_{2}\right)=1.5286$ at $\left.\lambda=1550 \mathrm{~nm}\right)$ for the bi-layers. Soft glass-based state-of-theart technologies (e.g., extrusion technique) should enable fabrication of such all-solid Bragglike fiber geometry with great precision and repeatability (Feng et al. 2005; Price et al. 2007). We make use of the well known Matrix method originally developed in our group (Thyagarajan et al. 1991) to study the modal characteristics of our chosen Bragg fiber-like structure with aperiodic claddings (as shown in Fig.1(b)). Here it is assumed that the low refractive index contrast involved in the chosen glasses should support linearly polarized modes.

To investigate the pulse reshaping issue in transversely chirped microstructured bandgap geometry, we have optimized a 2 meter long linearly tapered CBLF. This fiber length provides a decreasing group velocity dispersion (GVD) parameter in the normal dispersion regime to the propagating pulse through it. Due to introduction of chirp in the transverse i.e. radial direction, the individual cladding thickness in subsequent bi-layers varies in a linear fashion, and remains invariant over the fiber length. On the other hand, longitudinal tapering introduces a scaling of the fiber parameters from the input end to the output end of the fiber along its length in a linear manner. However, the optimized linear tapering ratio is different from the optimized linear chirp ratio for this wavelength specific application. The pulse evolution along the tapered dispersion decreasing CBLF has been modeled by solving the nonlinear Schrödinger equation (NLSE) through the symmetrized split-step Fourier method (Agrawal 2001)

$$
\frac{\partial u}{\partial z}+\frac{\alpha}{2} u+i \frac{\beta_{2}(z)}{2} \frac{\partial^{2} u}{\partial t^{2}}-\frac{\beta_{3}(z)}{6} \frac{\partial^{3} u}{\partial t^{3}}-i \gamma(z)|u|^{2} u=0
$$

where $u$ is the slowly varying pulse envelop and $\alpha$ is the loss parameter. Longitudinally varying GVD parameter, TOD parameter and nonlinear coefficient are respectively denoted as $\beta_{2}(\mathrm{z}), \beta_{3}(\mathrm{z})$, and $\gamma(\mathrm{z})$. We must mention here that the Eq. (2) ignores the terms which incorporate cross phase modulation, Raman and Brillouin gain effects. In Eq. (2), the longitudinally varying GVD parameter mimics the gain term in an amplifying medium (Latkin et al. 2007).

\section{Results}

For simulation of the propagation characteristics, the cladding of the sample CBLF is assumed to have 6 bi-layers surrounding the low index core. This fiber geometry provides a strong confinement of the fundamental $\mathrm{LP}_{01}$ mode at the designed operating wavelength of $1550 \mathrm{~nm}$. In this section, we will consider the analysis of modal characteristics of this particular mode to appreciate the effect of chirping. The thickness of the high-index cladding layer $\left(d_{1}\right)$, immediately adjacent to the $10 \mu \mathrm{m}$ core having refractive index $\left(n_{2}=1.5286\right)$ of the CBLF, is assumed to vary from $0.44 \mu \mathrm{m}$ to $0.66 \mu \mathrm{m}$ over a period of 6 bi-layers, while the width of the low index cladding layer $\left(d_{2}\right)$ is kept constant at $0.85 \mu \mathrm{m}$ all along the 6 bilayers. The so chosen cladding parameters results in $f_{1}=1.5$ and $f_{2}=1$ for the above-mentioned chirp rate (same as chirp factor). Spectral dependence of leakage loss for a CBLF (solid curve) and the corresponding ideal Bragg fiber (dotted curve) is shown in Fig. 3a. From this figure it could be seen that an enhancement of the PBG by as much as $\sim 55 \%$ could be achieved as compared to the estimated bandgap afforded by its counterpart Bragg fiber with perfectly periodic claddings. Simultaneously the central wavelength of the bandgap has been shifted from $1.2 \mu \mathrm{m}$ to $1.5 \mu \mathrm{m}$. This figure also indicates that the transmission window widens in the longer wavelength side of the band edge. It is worth mentioning that one could achieve 
enhancement of the bandgap on both the short and long wavelength band edges by continually varying the chirp factor starting with $f_{1}<1$ to $f_{1}>1$ as shown in Fig. 3b, where $f_{1}$ is varied from 0.8 to 1.5 . Similar characteristic is yielded by varying $f_{2}$ while keeping $f_{1}$ fixed. Thus using the same core as the original Bragg fiber and engineering the microstructured claddings through introduction of a suitable chirp, as described above we could design a $\mathrm{MOF}$, in which light flow could be controlled with greater freedom in its design as compared to an ideal Bragg fiber geometry. This enhancement of the stop-band could also be seen in its higher order bandgaps. It may be worthwhile to point out that in order to exploit these features for tailoring light propagation within the modified bandgap effectively, one needs to choose the chirp rate in a suitable manner for a particular higher order band gap (different from that corresponding to the fundamental gap). Introduction of even a small chirp factor leads to significant enhancement in the higher order band gap. Due to the nature of distributed Bragg resonances, CBLF exhibits lower wavelength selectivity for the guided light and hence would yield much less net dispersion than a corresponding ideal Bragg fiber. In Fig. 4 we have shown the variation of group velocity dispersion (GVD) with wavelength for a Bragg fiber and its equivalent CBLF counter part in which $f_{1}$ varies from 0.8 to 1.5 while $f_{2}=1$. It is apparent from the figure that there is a substantial decrease in net dispersion in the case of CBLF as compared to its Bragg fiber counterpart geometry. Dispersion in the CBLF is lower in magnitude throughout the band (shown as inset: Fig. 4) and it significantly reduces at the band-edges. This should be attractive from the point of view of propagation of short pulses with tens of femto-seconds $(f s)$ duration. Shorter temporal pulses have broader spectral width, which implies that they are significantly influenced by the higher order dispersion at the band edges. Reduced dispersion at the band edges of the CBLF would avoid this issue considerably. Thus from the fiber design point of view, wide photonic band gap along with low dispersion at the band edges should be attractive for such applications. This is indeed demonstrated in Fig. 5 wherein we depict temporal outputs of an input pulse of width $50 \mathrm{fs}$ after propagation through 1 meter long CBLF and corresponding result for propagation through its counterpart Bragg fiber of same length. Through further optimization with regard to appropriate choice of the chirp factor for a CBLF, one could achieve much less distortion in pulse propagation. This feature should be very useful in medical endoscopy that involves delivery of high energy pulses through an optical fiber for medical diagnostics. Moreover, elimination of dispersive nature in a bandgap guided structure is of immense practical importance from communication and signal processing points of view. Our designed fiber should be less prone to deformations during fabrication (compared to its counter part chirped bandgap guided PCF) because structurally it is of all-solid geometry and it should be useful in the near to mid-IR wavelength region due to the low loss characteristic of the chosen material systems. We may mention that introduction of refractive index chirp in place of a spatial chirp in the cladding layers also yielded very similar results. Thus our designed microstructured optical fiber should find applications in low-dispersive pulse propagation over a wide low loss band. Since Bragg resonances are not sufficiently strong for a particular frequency range due to the bi-layers being non-identical, the confinement losses are relatively higher in a CBLF compared to its Bragg fiber counterpart with same number of bi-layers. So, there is a trade off between dispersion elimination and leakage loss control. Increase in the number of bi-layers has been seen to also reduce overall confinement loss of a CBLF as expected (dispersion elimination is already achieved through the chirped cladding layers). It can be observed that at the transition frequencies from one stop band to the next (for the next cladding period), small kinks or ripples appear within the enhanced PBG of the CBLF. For a given chirp factor, these kinks or ripples in loss or dispersion spectra could be smoothened by increasing the number of bi-layers. This reduction is attributed to smoother bandgap transition over more number of bilayers. Similar features should be also attainable in an air-core CBLF. Various application-specific air-core Bragg fibers have been proposed in the literature (Fink et al, 1999; Dasgupta et al, 2005; Pal et al., 2005).

\section{Nonlinear pulse propagation in a CBLF}


Realizing the potential application of the bandgap engineering in the transverse direction of the microstructured fiber, we deliberately introduce a longitudinal uniform tapering to increase the degrees of freedom to manipulate the pulse propagation in this device. For this purpose, we have optimized a $7.5 \mu \mathrm{m}$ low index core CBLF (of the above pair of soft glasses) of $f_{1}$ varying from 0.98 to 1.1 over a period of 6 bi-layers and $f_{2}=1.0$ with a linear down taper ratio of 0.95 over 2 meter length of the fiber. The thicknesses of the high-index layer $\left(d_{1}\right)$ and low index layer $\left(d_{2}\right)$ in claddings are $0.5 \mu \mathrm{m}$ and $4.9 \mu \mathrm{m}$, respectively. Assuming the taper to be linear and considering the propagation of the fundamental mode, we probe the nonlinear pulse propagation through the fiber. The designed fiber, though multimoded at the operating wavelength, effectively functions as a single-mode fiber due to the large differential leakage loss suffered by its higher order modes (HOMs). For our design, the leakage losses for fundamental mode $\left(\mathrm{LP}_{01}\right)$ and first higher-order $\left(\mathrm{LP}_{11}\right)$ mode were found to be $\sim 0.02$ and $>10$ $\mathrm{dB} / \mathrm{m}$, respectively. Discrimination of other higher order modes is attributed to higher confinement loss and lower fractional power (besides the launching efficiency) inside the respective mode. Thus for this CBLF with a lesser extent taper, we require a 2 meter length to achieve an optimum pulse reshaping. The variation of dispersion parameters $\left(\beta_{2}\right.$ and $\left.\beta_{3}\right)$ and nonlinear parameter $(\gamma)$ along the length of this optimized design have been shown in Fig. 6 (a). In this figure, $\beta_{2}$ and $\beta_{3}$ show a slight deviation from a linear variation along the length tapered fiber. This nonlinear nature is definitely a signature of the dispersion modification due to a two-fold dispersion tailoring effect (tapering and chirping). Thus this particular chirped tapered Bragg-like fiber is a novel technique for significant dispersion tailoring along the fiber length, which is not easily attainable in a tapered conventional Bragg fiber (Dasgupta et al, 2007). On the other hand, this figure also indicates that nonlinear coefficient $\gamma$ varies almost linearly along the taper fiber. The variation is within the upper and lower limits of $\gamma \approx$ 15.2 to $13.1(\mathrm{~W} \mathrm{~km})^{-1}$, that are nearly one order of magnitude higher compared to that of the corresponding tapered silica Bragg fiber. This difference is attributable to the higher nonlinearity of the chosen soft glasses $\left(n_{2} \approx 2 \times 10^{-19} \mathrm{~m}^{2} / \mathrm{W}\right)$.

We exploit this feature to investigate feasibility of generating parabolic pulses in such a fiber. Such pulses are beneficial for high-power fiber lasers and amplifiers, supercontinuum generation, and all-optical signal regeneration because of their resistance to optical wave breaking (Anderson, et al. 1993; Tamura and M. Nakazawa, 1996), self-similarity in shape (Kruglov et al, 2002), and nearly linear chirp. In this optimized tapered 2 meter long CBLF sample, we study the evolution of a Gaussian input pulse (operating wavelength as $980 \mathrm{~nm}$ ) with a temporal width (FWHM) 0.5 ps and peak power $900 \mathrm{~W}$. After a detail numerical investigation, we obtain the pulse evolution along the fiber length as shown in Fig. 6 (b). From this figure, one can easily identify the transformation of the input Gaussian pulse to a parabolic intensity pulse after propagation through the fiber. The reshaping of an input Gaussian pulse to desired parabolic pulse is also evident from the plot of output pulse profile shown in Fig. 7 (a). The inset on the same plot shows the unique feature of linear chirp acquired over the duration of the generated parabolic pulse. Here we have assumed a lossless fiber without any TOD effect along its length. In order to test true parabolic nature of the pulse at the fiber output end, the power profile on a logarithmic scale is shown in Fig. 7 (b). This figure illustrates the top-hat nature with almost linear tail, which is a clear signature of a nearly parabolic pulse. Recent studies have shown that the presence of nonzero TOD along the tapered fiber has a detrimental effect on the generated parabolic pulse quality (Wei-Ci et al. 2008; Zhang et al. 2009). As the parabolic generation itself requires a finite normal dispersion at the operating wavelength with decreasing GVD along length, thus presence of nonzero TOD is unavoidable (Hirooka and Nakazawa 2004). Hence to generate high quality parabolic pulse one could exploit the generic dispersion elimination feature of a CBLF. Figure 8 clearly indicates that adverse effect of the TOD on the generated parabolic pulse is less prominent in a tapered CBLF than its normal Bragg fiber counterpart. In a defect free tapered Bragg fiber, if we incorporate the TOD effect, we have observed that the generated parabolic pulse is highly distorted in its shape around the peak of the pulse as well as the wings. Whereas, Fig. 8 also shows that the parabolic pulse generated in a tapered CBLF counterpart has a very little distortion around its peak with no noticeable change in the wings. 
We may mention that with further optimization, an improved design could efficiently maximize the elimination of the TOD effect as compared to a defect free Bragg fiber. Though the parabolic pulse is generated from the interplay of nonlinearity and dispersion, loss parameter has also an important role as the over all phenomena is fiber length dependent. In order to estimate effect of finite loss on the quality of generated parabolic pulse through a fixed length fiber, we have considered three different loss configurations e.g. no loss, $1 \mathrm{~dB}$ and $2 \mathrm{~dB}$ loss (including material and confinement losses). The generated parabolic pulses in the presence of loss slightly degrade in shape as shown in Fig. 9. Consquently it shows that there is a relative degradation of the pulse quality with a change in pulse shape, which is also evident from the inset of Fig. 9 showing that the time window of linearly varying chirp becomes narrower. Thus the device length dependent nonlinearity strength, dispersion and loss values play an important role in the process of parabolic pulse generation. Therefore to achieve an efficient good quality stable parabolic pulse, one needs to minimize the total loss along with TOD (eventually optimizing the device aspect ratio) of the proposed tapered CBLF. In this context it would be interesting to study the effect of peak power of the input pulse on the generated parabolic pulses. As evident from the Fig. 10, that an increasing peak power could eventually degrade the quality of generated parabolic pulses introducing a small kink either side of the characteristic central top-hat. This has also resulted a significant reduction of the time window over which the chirp follows a linear variation. We may mention here that we have deliberately chosen the all solid non-silica based fiber geometry to make it feasible to fabricate preform and then the draw the fiber using state of the art extrusion technique. It has already been reported (Feng et al. 2005; Feng et al. 2009) that the extrusion technique is mature enough to produce repeatable fiber geometries consists of the LLF1 and SF6 glasses with great precision and control (Price et al. 2007). If the core size is relatively large (we have maintained the bigger core size of $7.5 \mu \mathrm{m}$ radius), then it is even easier to control the thinner cladding layer thicknesses. It requires a proper scaling of the outer diameter of the structured preform with respect to the pre-designed set of fiber parameters. So even though the required thickness difference between two successive highindex layers in our design is sufficiently small, it is still possible to achieve individual thickness within tolerable limits to appreciate the effect of chirp. The fabrication of our designed 2 meter long tapered CBLF is feasible using the extrusion technique. We can also realize the proposed taper ratio along the fiber length with a suitable control of the drawing speed from it's perform (Bogatyrev et al. 1991) using a rig designed for fabricating tapered fibers through selective heating and pulling (Pal et al. 2003; Pal et al. 2007).

\section{Conclusions}

In conclusion, our designed chirped clad Bragg-like fiber geometry is a novel design tool to engineer bandgaps of microstructured optical fibers and waveguides with lower dispersion and moderate loss. We have shown the potentiality and suitability of our design tool for broadband low loss transmission while simultaneously maintaining low dispersion. We believe this technique could be gainfully exploited not only for widening of the photonic bandgap of such wave guiding structures but should be useful in tailoring dispersion properties for applications like super continuum generation and short pulse propagation. The proposed 2 meter long tapered CBLF has shown the better potential as a candidate for parabolic pulse generation by minimizing the TOD effect. Thus our proposal to exploit quasiperiodic claddings in an otherwise photonic band gap guided optical structure should prove to be a versatile tool with respect to designing of application-specific microstructured light guides. Moreover, the proposed technique of chirped cladding could be exploited as a design tool for an air core Bragg-like optical fiber targeted for propagation of short but high intensity optical pulses required for applications like biomedical optics. 
Acknowledgements Bishnu Pal, currently on leave of absence from IIT Delhi, acknowledges support of Centre National de la Recherche Scientifique (CNRS) France for hosting his stay at LPMC, Université de Nice-Sophia Antipolis, Nice. The work was partially supported by the ongoing Indo-UK Collaboration project on "Application specific Microstructured Optical Fibers" under the UK-India Education and Research Initiative (UKIERI) program. 


\section{References}

Agrawal, G. P.: Nonlinear Fiber Optics. Third ed., Academic, San Diego, CA (2001)

Anderson, D., Desaix, M., Karlsson, M., Lisak, M., Quiroga-Teixeiro, M. L.: Wave-breaking-free pulses in nonlinear-optical fibers. J. Opt. Soc. Am. B 10, 1185 (1993).

Bogatyrev, V. A., Bubnov, M. M., Dianov, E. M., Kurkov, A. S., Mamyshev, P. V., Prokhorov, A. M., Rumyantsev, S. D., Semenov, V. A., Semenov, S. L., Sysoliatin, A. A., Chernikov, S. V., Guryanov, A. N., Devyatykh, G. G., Miroshnichenko, S. I.: A single mode fiber with chromatic dispersion varying along the length. J. Lightwave Technol. 9, 561(1991)

Dasgupta, S., Pal, B. P., Shenoy, M. R.: Design of a Dispersion Compensating Bragg Fiber with ultrahigh Figure of merit. Opt. Letts. 30, 1917 (2005)

Dasgupta, S., Pal, B. P., Shenoy, M. R.: Nonlinear spectral broadening in solid core Bragg fibers. J. Lightwave Tech. 25, 2475 (2007)

Feng, X., Monro, T. M., Petropoulos, P., Finazzi, V., Richardson, D. J.: Extruded single mode highindex-core one-dimensional microstructured optical fiber with high index contrast for highly nonlinear optical deices. Appl. Phys. Lett. 87, 81110 (2005)

Feng, X., Poletti, F., Camerlingo, A., Parmigiani, F., Horak, P., Petropoulos, P., Loh, W. H., Richardson, D. J.: Dispersion-shifted all-solid high index-contrast microstructured optical fiber for nonlinear applications at 1.55 $\mu \mathrm{m}$. Opt. Exp. 17, 20249 (2009)

Fink, Y., Ripin, D. J., Fan, S., Chen, C., Joannopoulos, J. D., Thomas, E. L.: Guiding optical light in air using an all-dielectric structure. J. Lightwave Tech. 17, 2039 (1999)

Hirooka, T., Nakazawa, M.: Parabolic pulse generation by use of a dispersion-decreasing fiber with normal GVD. Opt. Lett. 29, 498 (2004)

Knight, J.C., Broeng, J., Birks, T. A., Russell, P. St. J.: Photonic bandgap guidance in optical fibers. Science 282, 1476 (1998)

Kruglov, V. I., Peacock, A. C., Harvey, J. D., Dudley, J. M.: Self-similar propagation of parabolic pulses in normal-dispersion fiber amplifiers. J. Opt. Soc. Am. B 19, 461 (2002)

Latkin, A.I., Turitsyn, S. K., Sysoliatin, A. A.: Theory of parabolic pulse generation in tapered fiber. Opt. Lett. 32, 331 (2007)

Li, J., Xue, L. J., Wang, Z., Han, Y. C.: Colloidal photonic crystals with a graded lattice-constant distribution. Colloid. Polym. Sci. 285, 1037 (2007)

Liu, B. Wen, Hu, M., Fang, X. H., Li, Y. F., Chai, L., Li, J. Y., Chen, W., Wang, C. Y.: Tunable bandpass filter with solid core photonic bandgap fiber and Bragg fiber. IEEE Photon. Technol. Lett. 20, 581 (2008)

Millo, A., Lobachinsky, L., Katzir, A.: Single-mode index-guiding photonic crystal fibers for the middle infrared. Photon. Technol. Lett. 20, 10, 869 (2008)

Mori, D., Baba, T.: Dispersion-controlled optical group delay device by chirped photonic crystal waveguides. Appl. Phys. Lett. 85, 1101 (2004)

Nagaraju, B., Varshney, R. K., Agrawal, G. P., Pal, B. P.: Parabolic pulse generation in a dispersion decreasing solid core photonic bandgap Bragg fiber. Opt. Comm. 283, 2525 (2010)

Nielsen, C. K., Jespersen, K. G., Keiding, S. R.: A $158 \mathrm{fs} 5.3 \mathrm{~nJ}$ fiber-laser system at $1 \mu \mathrm{m}$ using photonic bandgap fibers for dispersion control and pulse compression. Opt. Express 14, 6063 (2006)

Pal, B. P., Roy Chaudhuri, P., Shenoy, M. R.: Fabrication and modeling of fused biconical tapered fiber coupler components. Fiber Int. Opt. 22, 97 (2003)

Pal, B. P., Dasgupta, S., Shenoy, M.R.: Bragg Fiber Design for Transparent Metro Networks. Opt. Exp. $13,621(2005)$

Pal, B. P., Dasgupta, S., Shenoy, M. R., Sysoliatin, A.: Supercontinuum generation in a Bragg fiber: a novel proposal. Optoelectron. Lett. 2, 342 (2006)

Pal, B. P., Ghosh, S., Varshney, R. K., Dasgupta, S., Ghatak, A.: Loss and dispersion tailoring in 1D photonics bandgap Bragg reflection waveguides: finite chirped claddings as a design tool. Opt. and Qua. Elec. 39, 983 (2007)

Pal, P., Knox, W. H.: End-sealing short dispersion micromanaged tapered holey fibers by holecollapsing. Opt. Exp. 15, 13531 (2007)

Pal, B. P., Ghosh, S., Varshney, R. K.: Microstructured fibers and waveguides with a chirped cladding: a new versatile design platform to enhance their functionality. International conference on optics and photonics (ICOP-2009). $30^{\text {th }}$ Oct- $1^{\text {st }}$ Nov, Chandigarh, India (2009)

Price, J. H. V., Monro, T. M., Ebendorff-Heidepriem, H., Poletti, F., Horak, P., Finazzi, V., Leong, J. Y. Y., Petropoulos, P., Flanagan, J. C., Brambilla, G., Feng, X. Richardson, D. J.: Mid-IR supercontinuum generation from nonsilica microstructured optical fibers. Sel. Topic in Quan. Elecrton. 3, 738 (2007)

Ranka, J. K., Windeler, R. S., Stentz, A. J.: Visible continuum generation in air-silica microstructure optical fibers with anomalous dispersion at $800 \mathrm{~nm}$. Opt. Lett. 25, 25 (2000)

Russell, P.: Photonic crystal fibers. Science 299, 358 (2003) 
Saitoh, K., Koshiba, M., Hasegawa, T., Sasaoka, E.: Chromatic dispersion control in photonic crystal fibers: application to ultra-flattened dispersion. Opt. Express 11, 843 (2003)

Skibina, J. S., Iiew, R., Bethge, J., Bock, M., Fischer, D., Beloglasov, V. I., Wedell, R., Steinmeyer, G.: A chirped photonic crystal fiber. Nature Photonics 2, 679 (2008)

Tamura, K. Nakazawa, M.: Pulse compression by nonlinear pulse evolution with reduced optical wave breaking in erbium-doped fiber amplifiers, Opt. Lett., 21, 68 (1996)

Temelkuran, B., Hart, S. D., Benoit, G., Joannopoulos, J. D., Fink, Y.: Wavelength-scalable hollow optical fibres with large photonic bandgaps for $\mathrm{CO}_{2}$ laser transmission. Nature 420, 650 (2002)

Thyagarajan, K., Diggavi, S., Taneja, A., Ghatak, A. K.: Simple numerical technique for the analysis of cylindrically symmetric refractive-index profile optical fibers. Appl. Opt. 30, 3877 (1991)

Wei-Ci, L., Wen-Cheng, X., Jie, F., Wei-Cheng, C., Shu-Xian, L., Song-Hao, L.: Higher order effects on self similar parabolic pulse in the microstructured fiber amplifier. Chinese Phys. B, 17, 1025 (2008)

Zhang, S., Zhao, G., Luo, A., Zhang, Z.: Third order dispersion role on parabolic pulse propagation in dispersion decreasing fiber with normal group velocity dispersion. Appl. Phys. B, 94, 227 (2009) 


\section{Figures:}

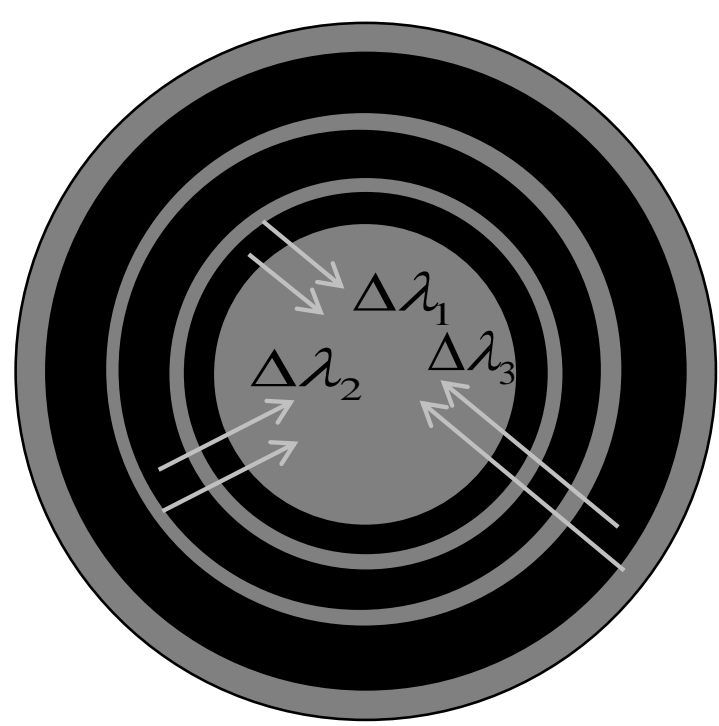

Fig. 1(a). Schematic diagram of a chirped Bragg like fiber geometry in which inherent distributed Bragg resonances within its claddings essentially leads to widening of the photonic bandgap.

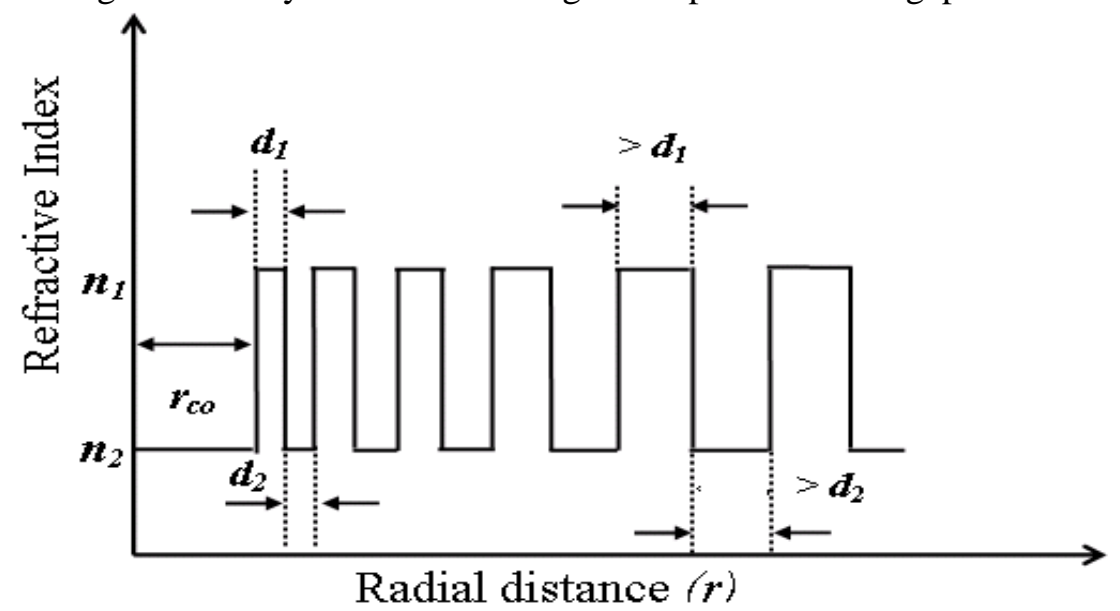

Fig. 1(b). Schematics of the refractive index profile (RIP) of the proposed chirped Bragg-like fiber (CBLF).

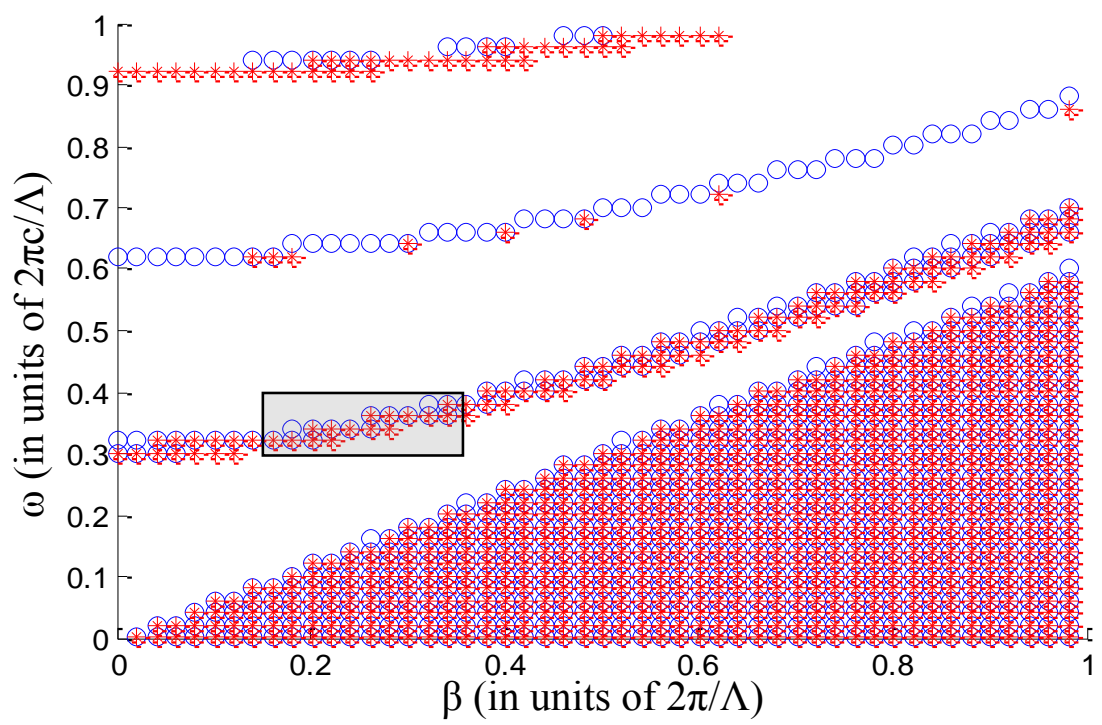

Fig. 2(a). Band diagram plotted here for two different cladding periods; first one: $d_{1}=0.44 \mu \mathrm{m}, d_{2}=0.85 \mu \mathrm{m}$ ('blue circles (o)'), second one: $d_{1}=0.66 \mu \mathrm{m}, d_{2}=0.85 \mu \mathrm{m}$ ('red stars $\left({ }^{*}\right)$ '). In both the cases, we have assumed the periodicity is invariant over infinite cladding. 


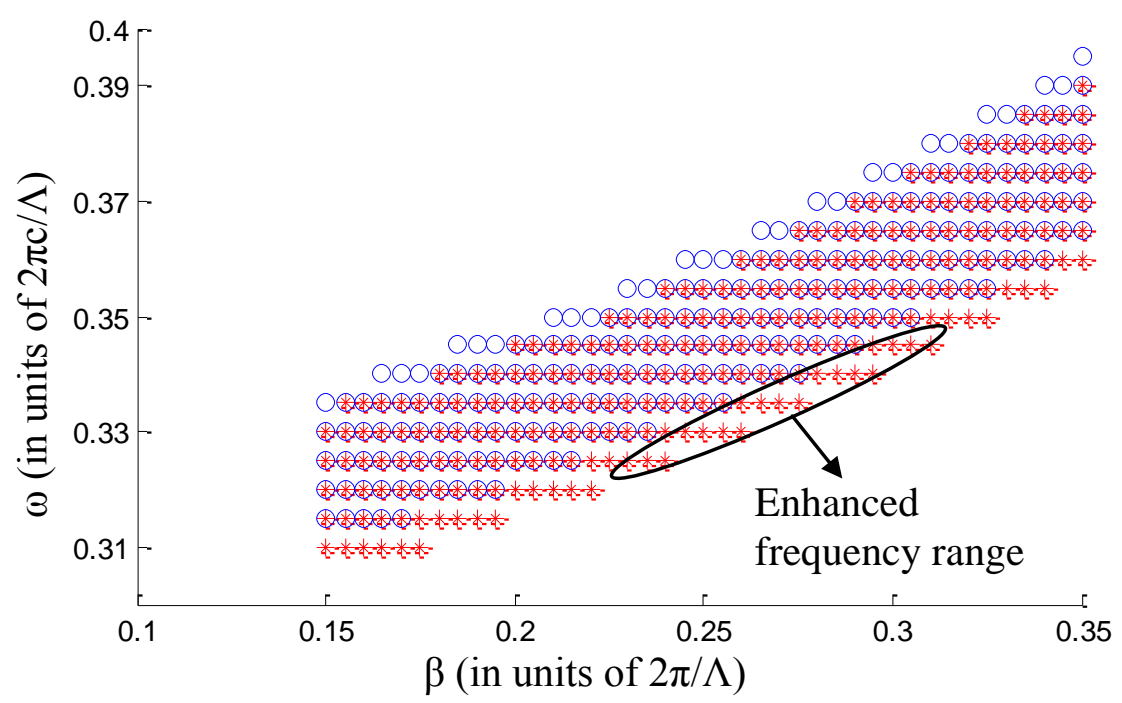

Fig. 2(b). A zoomed view over a narrower frequency window of the fundamental bandgap which is shown in Fig. 2(a) (shaded box area).

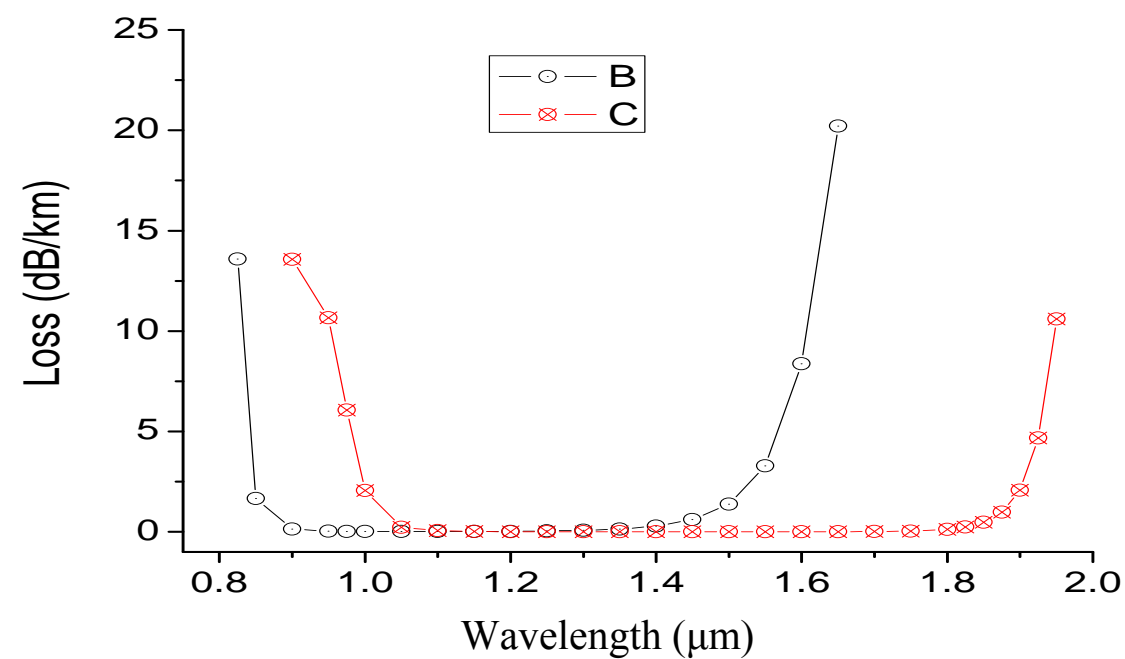

Fig. 3(a). Numerically simulated loss spectra, B: original Bragg fiber, C: its CBLF counterpart. 


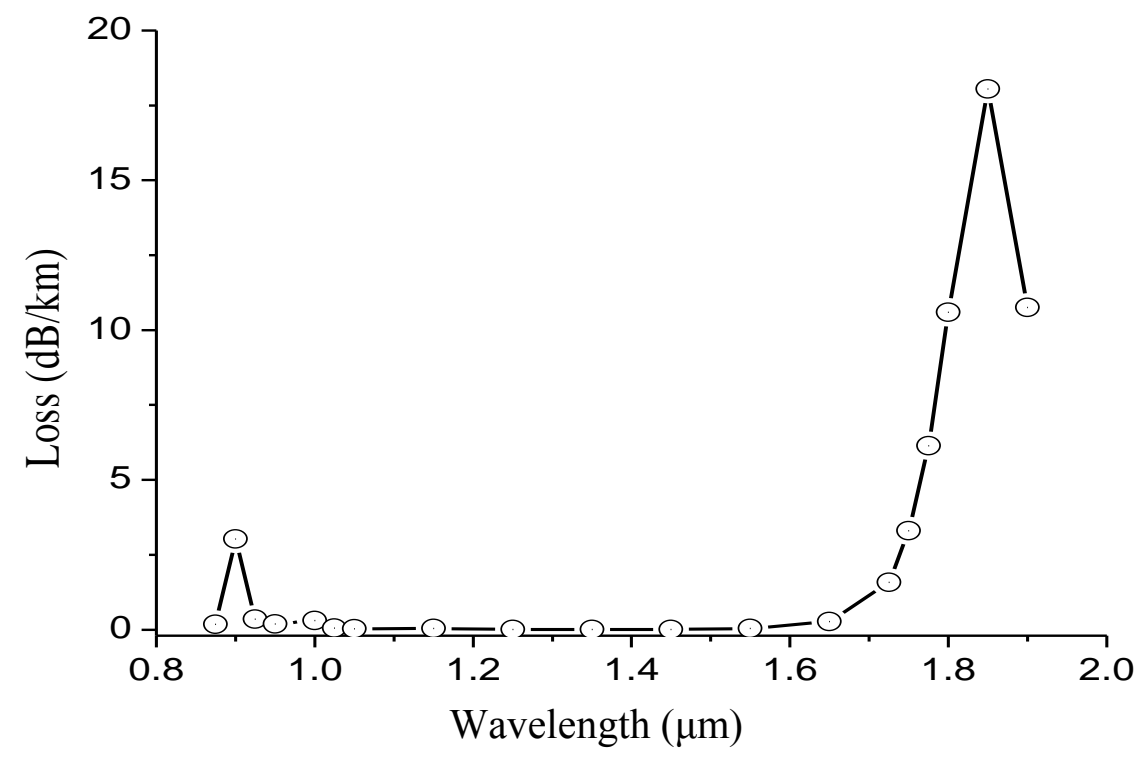

Fig. 3(b). Low loss broadband transmission using a CBLF with 8 bilayers, in which $f_{1}$ varies continuously from 0.8 to 1.5 and $f_{2}=1$ ).

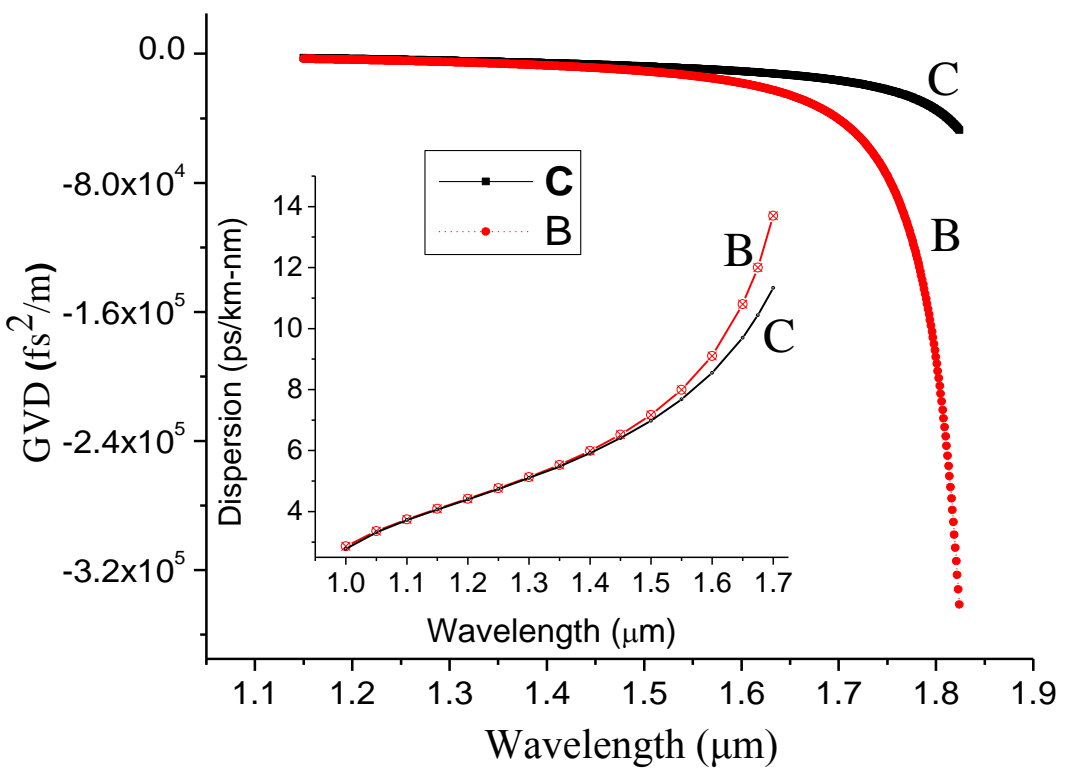

Fig. 4. Spectral dependence of GVD; Solid black curve-C: for a CBLF, Dotted red curve-B: for its counterpart Bragg fiber. Inset: Dispersion behavior of the Bragg fiber (B) and its CBLF counterpart. 


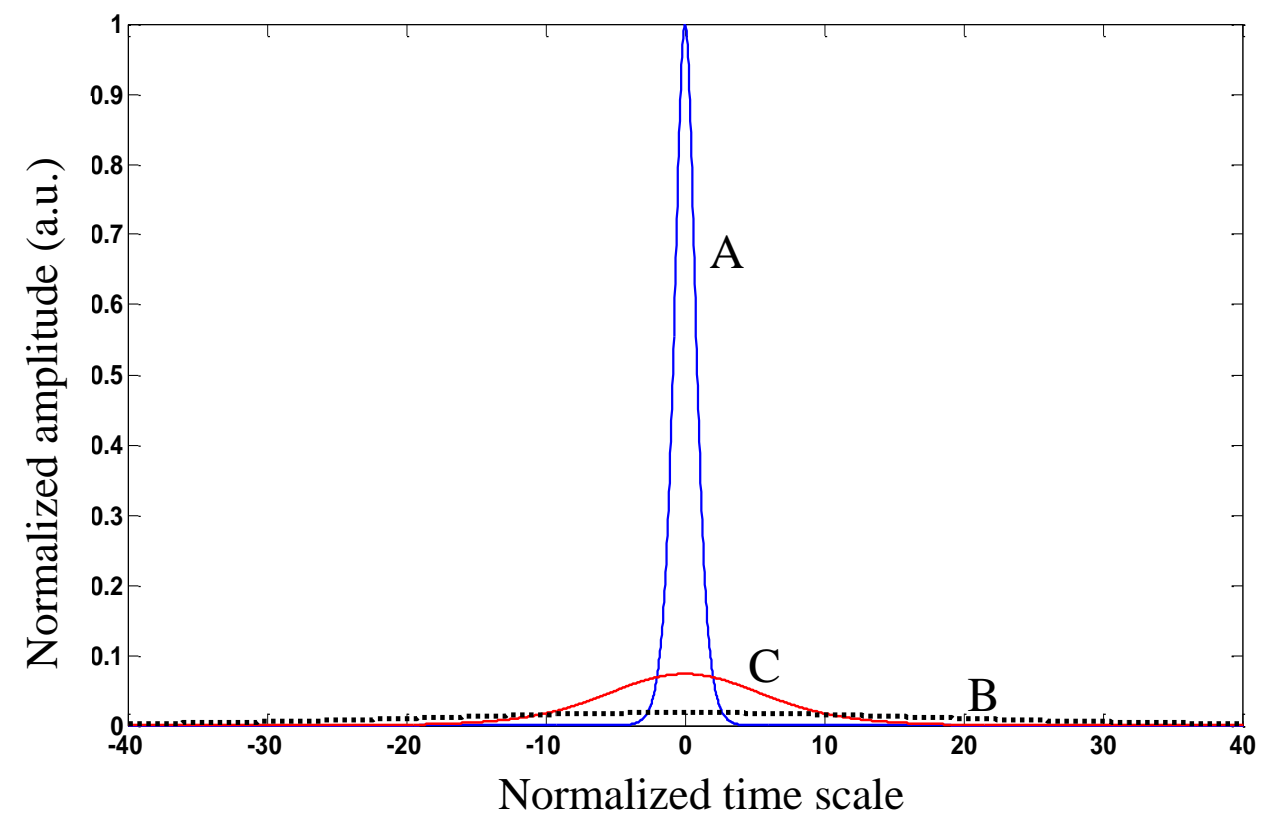

Fig. 5. Output pulses relative to an input pulse (shown as solid blue curve 'A') of width $50 \mathrm{fs}$ after propagation through 1 meter long fiber; where solid red curve (' $\mathrm{C}$ ') represents output pulse from the CBLF and dotted black curve ('B') represents output pulse from its counterpart Bragg fiber.
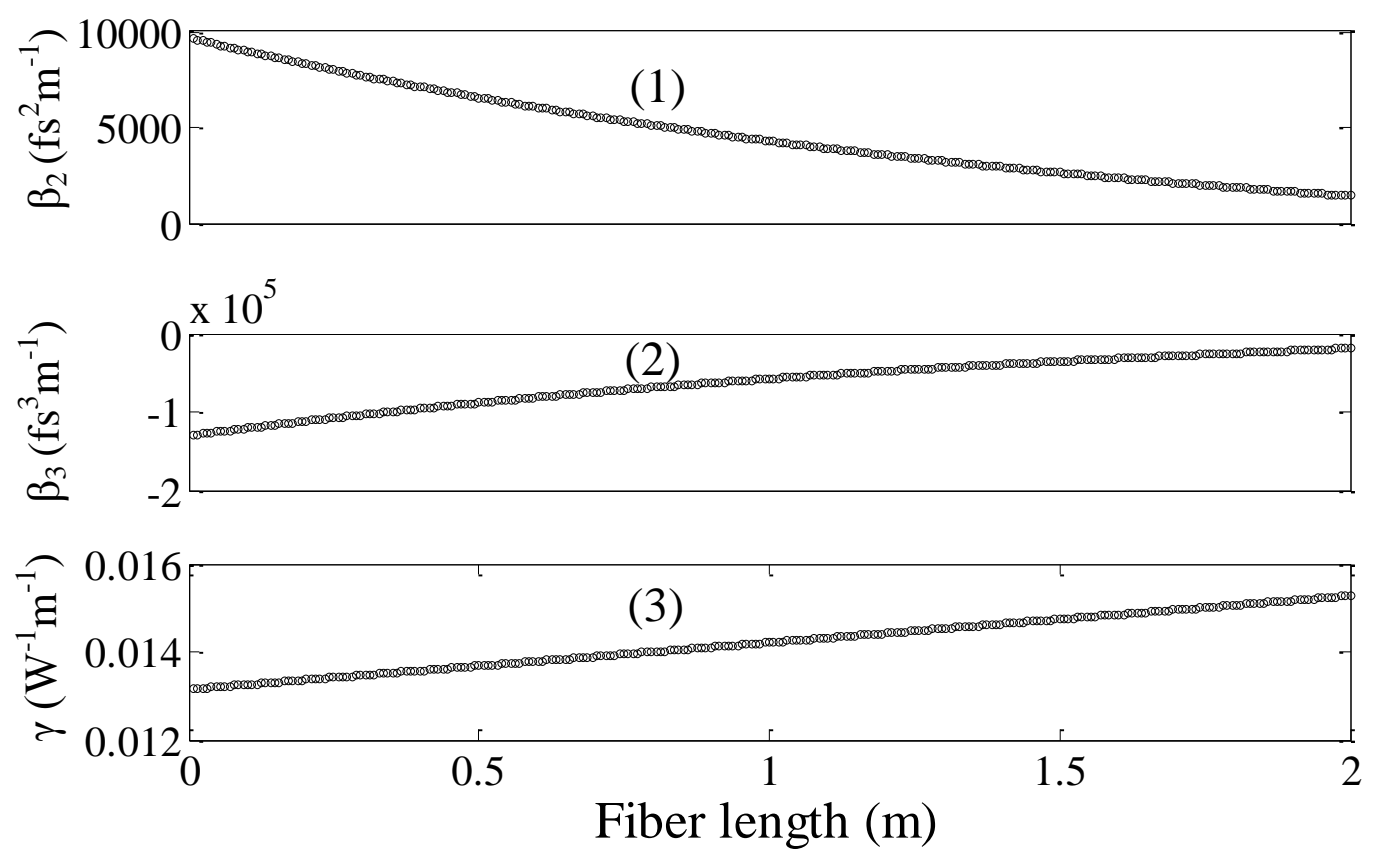

Fig. 6(a). Variation of GVD parameter (subplot-1), TOD coefficient (subplot-2) and nonlinearity parameter (subplot-3) along the proposed tapered CBLF length. 


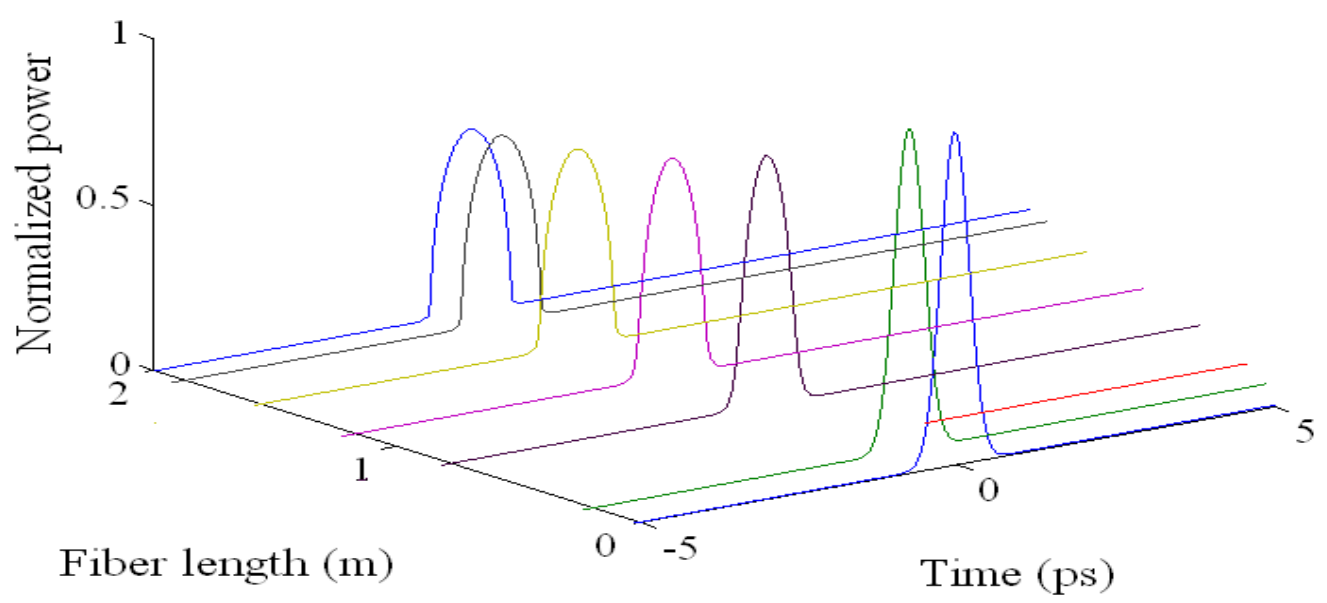

Fig. 6(b). Evolution of an input Gaussian pulse to a parabolic pulse with propagation in a lossless 2 meter long proposed tapered CBLF.

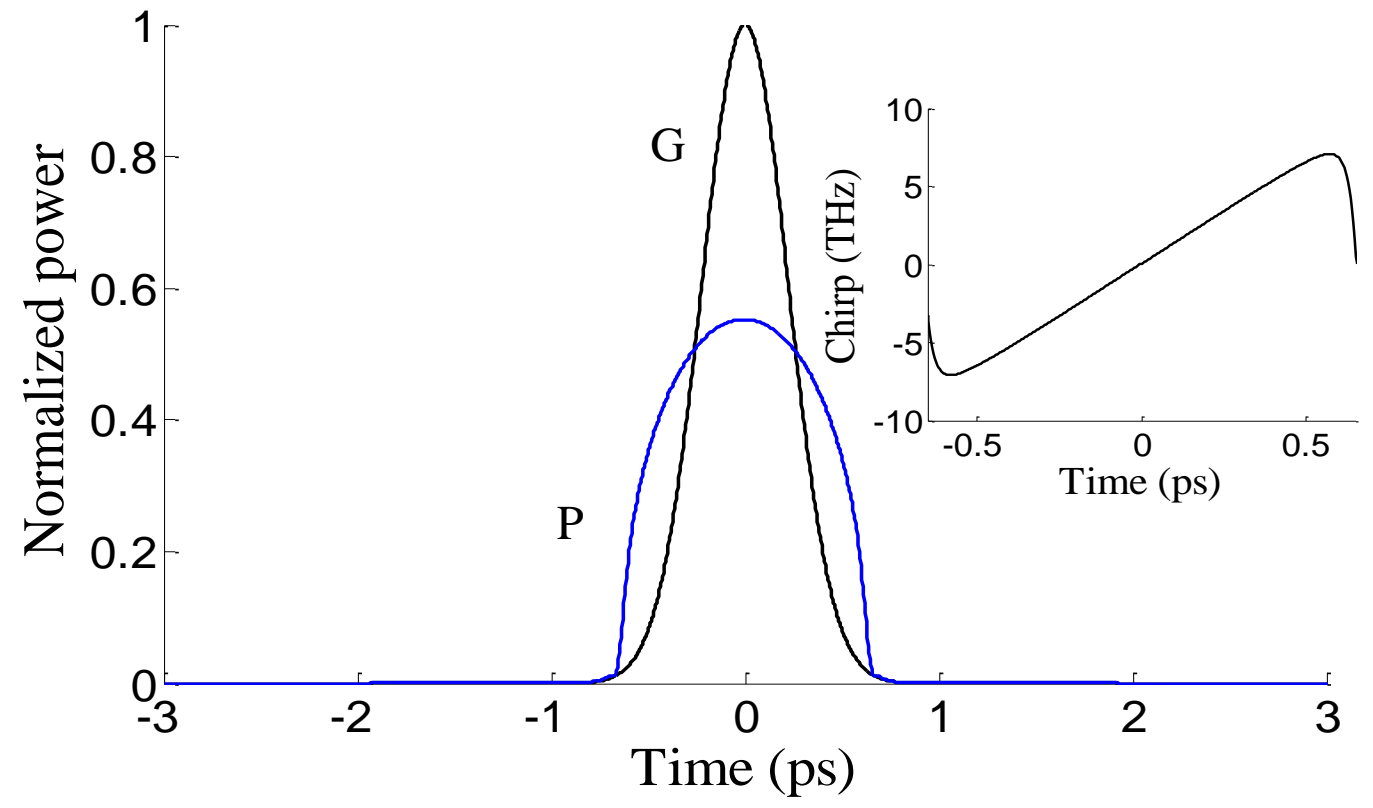

Fig. 7(a) 


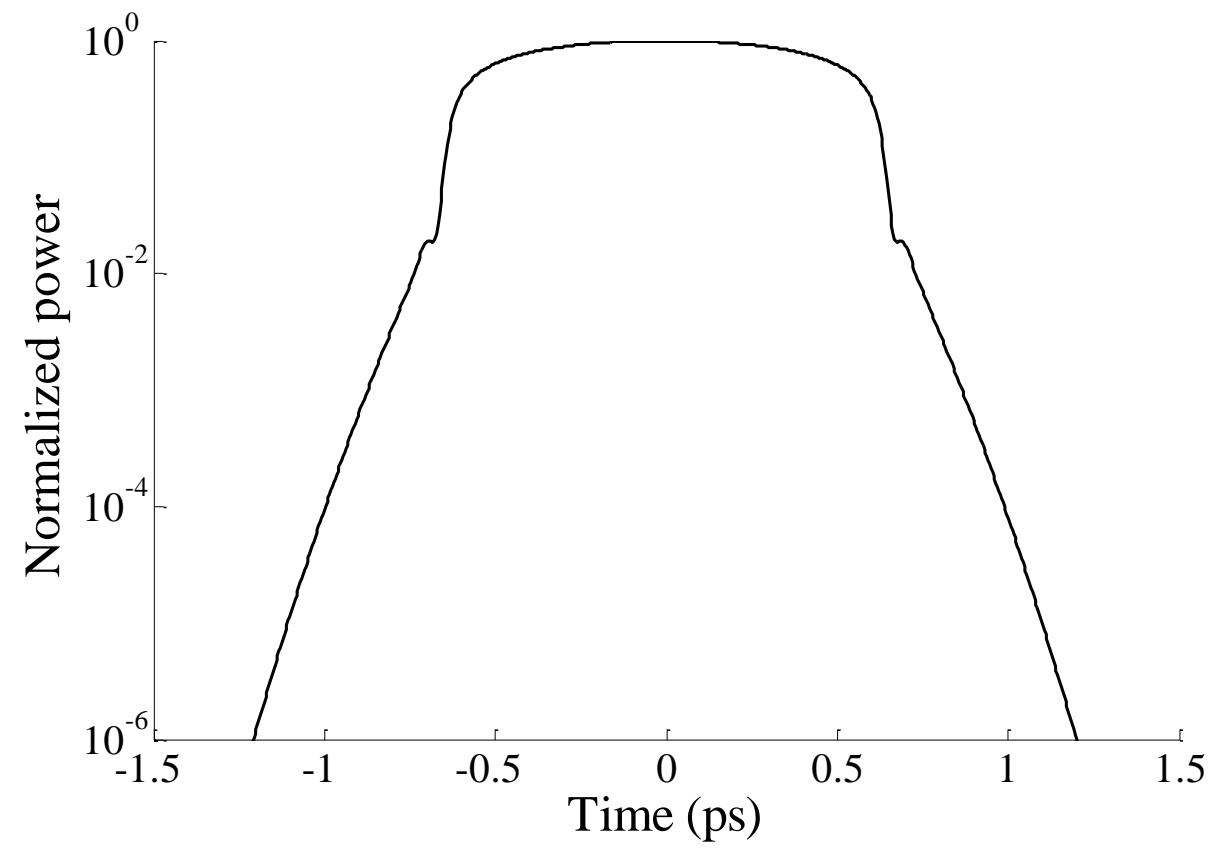

Fig. 7(b)

Fig. 7. Output pulse shape from the proposed 2 meter long lossless tapered CBLF design. (a) in linear scale ('G' - input Gaussian pulse, 'P' - output parabolic pulse), inset shows the corresponding chirp variation; (b) in logarithmic scale.

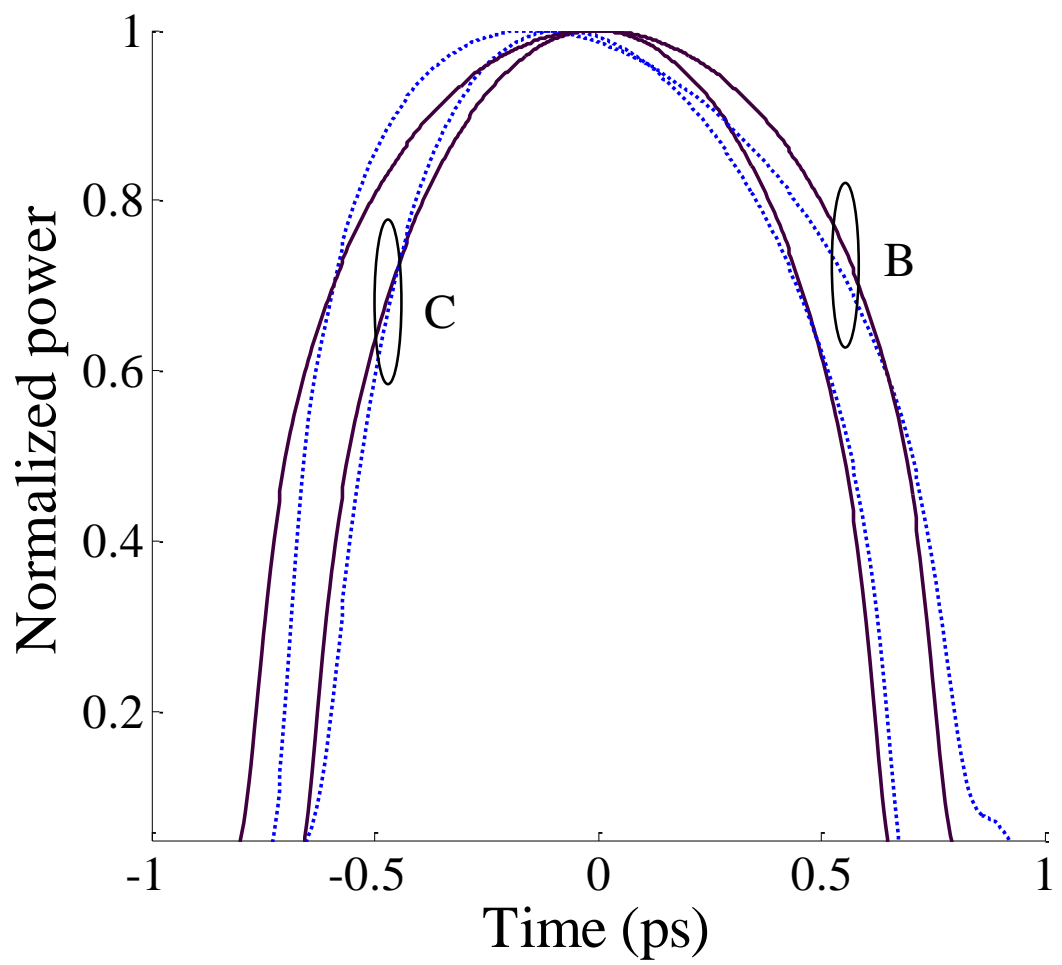

Fig. 8. Generated parabolic pulses with (dotted curve) and without (solid curve) TOD effect in Bragg fiber (curve-B) and CBLF (curve-C). In both the cases, input pulse power is always same (850W). 


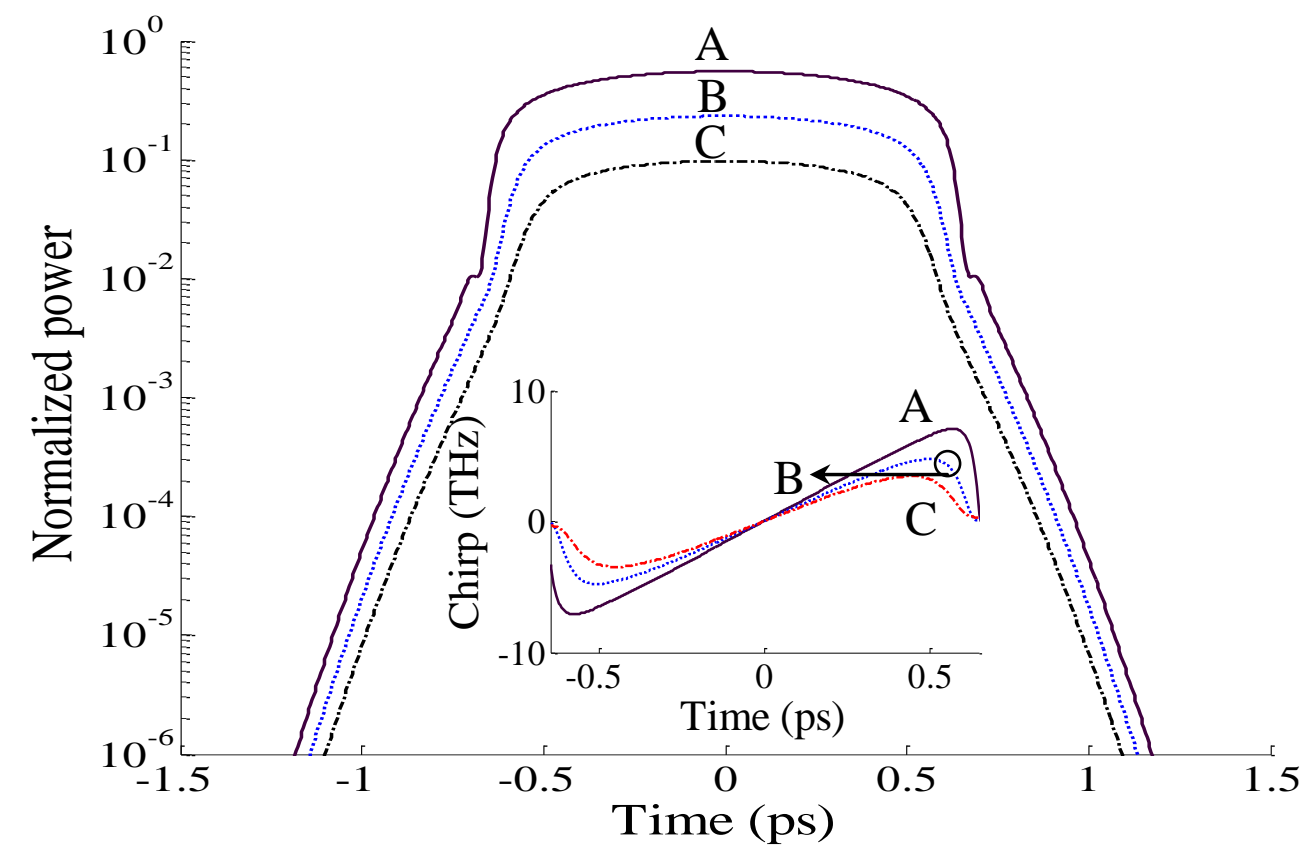

Fig. 9.

Fig. 9. Generated parabolic pulse from the tapered CBLF for three different cases, namely without loss (solid curve ' $A$ '), $1 \mathrm{~dB}$ loss (dotted curve 'B'), and $2 \mathrm{~dB}$ loss (dash-dotted curve ' $C$ '); Inset: Chirp variations over the pulse duration corresponding to the above three respective configurations. 


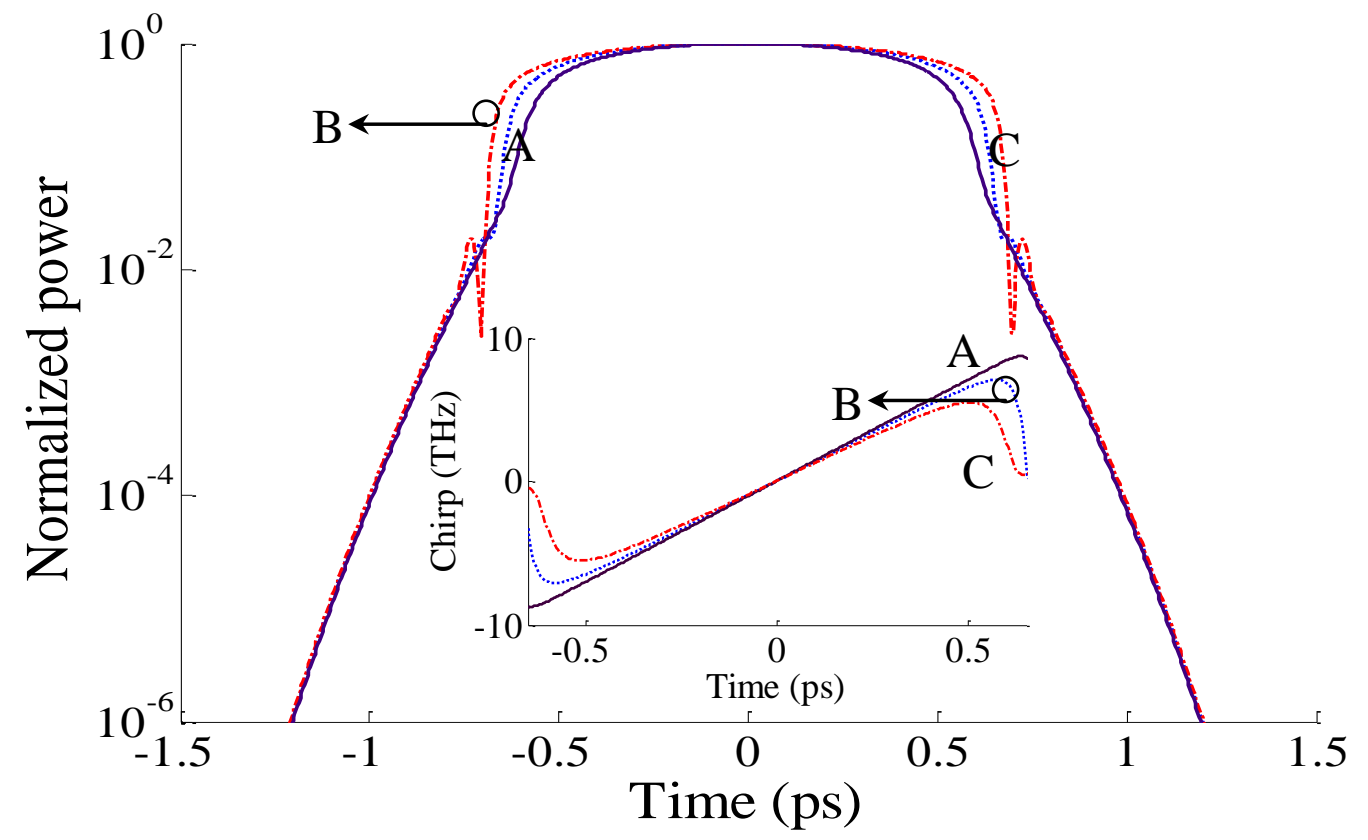

Fig.10

Fig. 10 Generated parabolic pulse from the tapered CBLF for three different peak powers of input pulse, 600 W peak power (solid curve 'A'), 800W peak power (dotted curve 'B'), and 1000W peak power (dash-dotted curve ' $\mathrm{C}$ '); Inset: Chirp variations over the pulse duration corresponding to the above three respective input conditions. 